\title{
Effects of distraction task on driving: A functional magnetic resonance imaging study
}

\author{
Soon-Cheol Chung ${ }^{a}$, Mi-Hyun Choi ${ }^{\text {a }}$, Hyung-Sik Kim ${ }^{a}$, Na-Rae You ${ }^{a}$, Sang-Pyo Hong ${ }^{\text {a }}$, \\ Jung-Chul Lee ${ }^{\mathrm{a}}$, Sung-Jun Park ${ }^{\mathrm{a}}$, Ji-Hye Baek ${ }^{\mathrm{a}}$, Ul-Ho Jeong ${ }^{\mathrm{a}}$, Ji-Hye You ${ }^{\mathrm{a}}$, Dae-Woon Lim ${ }^{\mathrm{b}}$ \\ and Hyun-Jun Kim, ${ }^{\text {c, }}$ \\ ${ }^{a}$ Department of Biomedical Engineering, Research Institute of Biomedical Engineering, College of \\ Biomedical \& Health Science, Konkuk University, Chungju 380-701, South Korea \\ ${ }^{b}$ Department of Information \& Communication Engineering, Dongguk University, Seoul 100-715, \\ South Korea \\ ${ }^{c}$ Department of Obstetrics \& Gynecology, Konkuk University, Chungju 380-701, South Korea
}

\begin{abstract}
This study investigated neuronal activation differences under two conditions: driving only and distracted driving. Driving and distraction tasks were performed using a Magnetic Resonance (MR)-compatible driving simulator with a driving wheel and pedal. The experiment consisted of three blocks, and each block had both a Rest phase (1 min) and a Driving phase $(2 \mathrm{~min})$. During the Rest phase, drivers were instructed to simply look at the stop screen without performing any driving tasks. During the Driving phase, each driver was required to drive at $110 \mathrm{~km} / \mathrm{h}$ under two conditions: driving only and driving while performing additional distraction tasks. The results show that the precuneus, inferior parietal lobule, supramarginal gyrus, middle frontal gyrus, cuneus, and declive are less activated in distracted driving than in driving only. These regions are responsible for spatial perception, spatial attention, visual processing and motor control. However, the cingulate gyrus and sub-lobar regions (lentiform nucleus and caudate), which are responsible for error monitoring and control of unnecessary movement, show increased activation during distracted driving compared with driving only.
\end{abstract}

Keywords: Distraction, driving, fMRI, MR-compatible driving simulator

\section{Introduction}

A number of studies have shown that neural activities are associated with driving using functional magnetic resonance imaging (fMRI) [1-11]. When driving by using a computer mouse and trackball, the parietal cortex, occipital cortex, motor cortex, and cerebellum were all activated [2]. Uchiyama et al. had participants use a joystick to control vehicles and observed activation of the superior and inferior parietal lobules, superior, middle and inferior frontal gyri, middle temporal gyrus, primary sensorimotor cortex, basal ganglia, supplementary motor region, and cerebellum. When a driving

\footnotetext{
${ }^{*}$ Corresponding author: Hyun-Jun Kim, Department of Obstetrics \& Gynecology, Konkuk University, Chungju 380-701, South Korea. Tel.: +82-17-258-2192; Fax:+82-43-851-0620; E-mail: icarus@kku.ac.kr.
} 
scene was simply observed, the superior temporal gyrus, middle and inferior frontal gyri, occipital lobe, orbitofrontal cortex, cingulate gyrus, fusiform gyrus, supplementary motor region, and basal ganglia regions were all activated [11].

Additional tasks, such as dialing mobile phones, tuning the radio, eating, or having a conversation, occur frequently during driving. fMRI studies have elucidated the brain responses associated with driving while performing distraction tasks, such as auditory language comprehension and visual event detection $[1,2,5-7,9]$. The activated regions in distracted driving were similar to those in driving only, but these regions showed decreased activation when distracted driving occurred.

It is important to note that these previous studies used computer mice, trackballs or joysticks to simulate driving, which are not comparable to real driving situations. This study used a Magnetic Resonance (MR)compatible driving simulator with a driving wheel and pedal in order to overcome the limitations of the previous studies. Our purpose was to determine whether there are any differences between the neural activities elicited during driving only and distracted driving, using a driving simulator that could depict scenes closer to reality.

\section{Methods}

Sixteen male drivers (age $26.6 \pm 2.1 \mathrm{y}$; driving experience $2.7 \pm 1.5 \mathrm{y}$ ) without any psychiatric illness or nerve/brain-related conditions participated in this study. Prior to the experiment, informed consent was obtained from each participant, and the purpose and content of the experiment were explained. The experimental design controlled all external factors (e.g., smoking, alcohol, and coffee consumption) that could influence driving performance. The experiment was conducted at the time when participants felt they would have no difficulty in using the driving simulator.

An MR-compatible driving simulator with a driving wheel and pedal (Figure 1) was developed. The driving environment was simulated using the software provided by Lightrock Entertainment (Leipzig, Germany), and was mostly made up of "straight road"-like areas with very few distracting elements. The subjects used both hands to operate the wheel and used their right foot to control the accelerator and brake. They were instructed to drive without changing lanes at a constant speed $(110 \mathrm{~km} / \mathrm{h})$. The visual information of their driving was shown on a visual system attached to the subject' head coil.
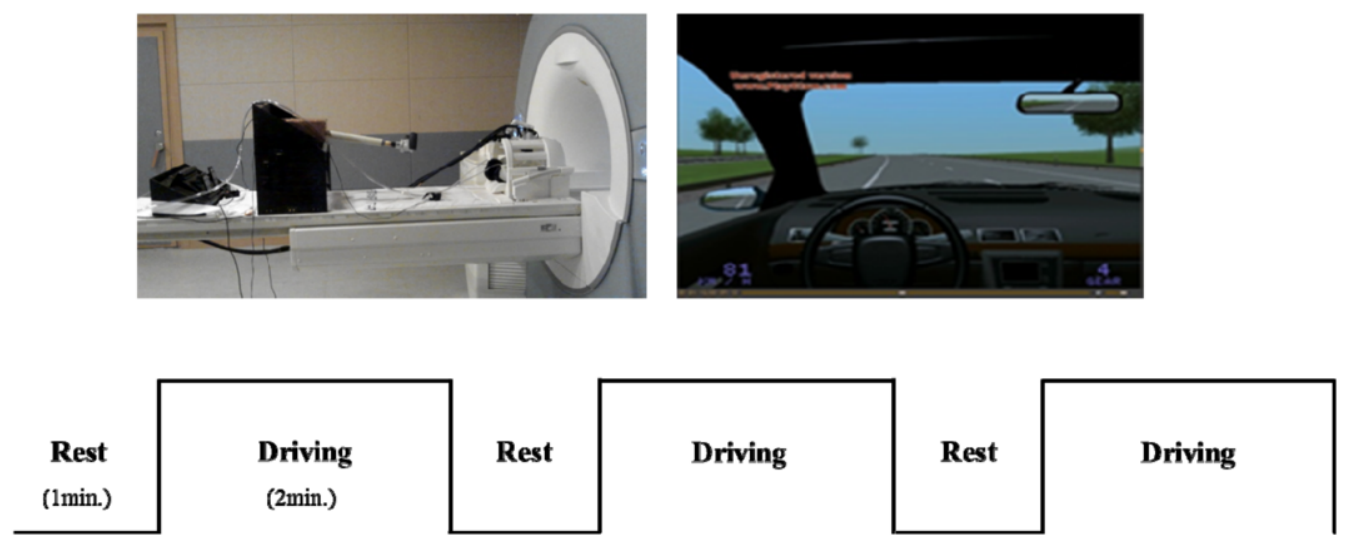

Fig. 1. MR-compatible driving simulator with driving wheel and pedals (accelerator and brake), and a schematic of the fMRI experimental procedure. 
The experiment was composed of three blocks (Figure 1). Each block consisted of a Rest phase (1 min) and a Driving phase (2 min). During the Rest phase, drivers were instructed to look at the screen, which gave them an experience of being in a parked, non-moving state. During the Driving phase, drivers were required to either focus on driving (Driving Only, DO) or drive while performing a distraction task (Driving With Task, DWT), at $110 \mathrm{~km} / \mathrm{h}$. Each subject participated in both tasks (DO and DWT). The order of experiments was counterbalanced. The speed of the vehicle was displayed in the lower left-hand corner of each subject's screen in order to help them maintain a constant speed of $110 \mathrm{~km} / \mathrm{h}$.

An additional task was selected for distracted driving, that is, double-digit carry-over calculation with sums less than 100. Each block in the additional task comprised 10 questions, with a total of 30 questions in three blocks. The experimenters used an audio system attached to the MRI machine to present the task instructions; subjects confirmed the answers vocally. Subjects were encouraged to concentrate on both driving and additional tasks.

Imaging was conducted on a 3T MRI system (Magnetom TrioTim, Siemens Medical Systems, Erlangen, Germany) equipped with a standard 32-channel head coil. Single-shot echo planar fMRI scans were acquired in 29 continuous slices, parallel to the anterior commissure-posterior commissure line. The parameters for fMRI were as follows: TR/TE $=3000 / 30 \mathrm{~ms}, \mathrm{FOV}=200 \mathrm{~mm}$, flip angle $=$ $90^{\circ}$, matrix $=128 \times 128$, slice thickness $=5 \mathrm{~mm}$, voxel size $=1.6 \mathrm{~mm} \times 1.6 \mathrm{~mm} \times 5.0 \mathrm{~mm}$. T1weighted anatomical images were obtained with a 3D MPRAGE sequence (TR/TE $=1900 / 2.48 \mathrm{~ms}$, $\mathrm{FOV}=200 \mathrm{~mm}$, flip angle $=9^{\circ}$, matrix $=256 \times 256$, slice thickness $=1 \mathrm{~mm}$, voxel size $=0.8 \mathrm{~mm} \times$ $0.8 \mathrm{~mm} \times 1.0 \mathrm{~mm})$.

fMRI data were analyzed with SPM 8 software (Wellcome Department of Cognitive Neurology, London, UK). All functional images were aligned with the anatomical images using affine transformation routines built into the SPM 8 program. The realigned scans were co-registered to the participant's anatomical images, which were obtained within each session, and then normalized to a template image in SPM 8. This software uses the space defined by the Montreal Neurologic Institute, which is very similar to Talairach and Tournoux's [12] stereotaxic atlas. Motion correction was performed using sinc interpolation. Time-series data were filtered with a 240 -s high-pass filter in order to remove artifacts due to cardiorespiratory and other cyclical influences. The functional map was smoothened with an 8-mm isotropic Gaussian kernel prior to statistical analysis. Statistical analyses were performed both individually and as a group using a general linear model and the theory of Gaussian random fields implemented in SPM 8.

The active regions of the brain during DO and DWT periods compared with the Rest phase were extracted using the subtraction method (DO or DWT-Rest). Finally, the double subtraction method was used to observe any regions exhibiting contrast activity under both driving conditions (DO-DWT and DWT-DO).

\section{Results}

Table 1 and Figure 2(a) show the activated regions from the "DO-DWT" contrast, i.e., brain regions that were activated under only driving were subtracted from the activated regions under distracted driving. The results reveal increased activation in the frontal (middle, inferior, medial, and superior frontal gyri and precentral gyrus), parietal (superior and inferior parietal lobules, postcentral gyrus, supramarginal gyrus, and precuneus), temporal (superior temporal gyrus), occipital (cuneus), sub-lobar (thalamus), limbic (parahippocampal gyrus) and cerebellar (declive, culmen, and cerebellar tonsil) 
Table 1

MNI coordinates, t-scores, and number of voxels in activated regions determined by the double subtraction method (DODWT) (corrected $\mathrm{p}<0.05$ )

\begin{tabular}{|c|c|c|c|c|c|}
\hline \multirow{3}{*}{$\begin{array}{l}\text { Number of voxels } \\
2712\end{array}$} & \multirow{3}{*}{$\frac{\text { t-score }}{10.43}$} & \multicolumn{3}{|c|}{ MNI coordinates } & \multirow[b]{3}{*}{ Precuneus } \\
\hline & & \multicolumn{2}{|c|}{$(\mathrm{x}, \mathrm{y}, \mathrm{z}(\mathrm{mm}))$} & Side & \\
\hline & & 11 & $-75 \quad 50$ & $\mathrm{R}$ & \\
\hline 120 & 7.69 & 51 & $8 \quad 35$ & $\mathrm{R}$ & Middle Frontal Gyrus \\
\hline 69 & 7.27 & 64 & $-31 \quad 40$ & $\mathrm{R}$ & Inferior Parietal Lobule \\
\hline 43 & 4.95 & 58 & $14 \quad 30$ & $\mathrm{R}$ & Inferior Frontal Gyrus \\
\hline 23 & 5.35 & 55 & 110 & $\mathrm{R}$ & Superior Temporal Gyrus \\
\hline 19 & 6.24 & 27 & $-7 \quad 65$ & $\mathrm{R}$ & Superior Frontal Gyrus \\
\hline 12 & 5.46 & 56 & $-26 \quad 50$ & $\mathrm{R}$ & Postcentral Gyrus \\
\hline 5 & 6.3 & 19 & $\begin{array}{ll}-26 & 5\end{array}$ & $\mathrm{R}$ & Thalamus \\
\hline 5 & 5.01 & 17 & 365 & $\mathrm{R}$ & Medial Frontal Gyrus \\
\hline 2961 & 11.39 & -22 & $-84 \quad 30$ & $\mathrm{~L}$ & Cuneus \\
\hline 329 & 7.05 & -55 & $-36 \quad 45$ & $\mathrm{~L}$ & Inferior Parietal Lobule \\
\hline 188 & 8.69 & -56 & $-37 \quad 30$ & $\mathrm{~L}$ & Supramarginal Gyrus \\
\hline 70 & 7.65 & -28 & $-50 \quad 40$ & $\mathrm{~L}$ & Superior Parietal Lobule \\
\hline 54 & 7.37 & -55 & $0 \quad 35$ & $\mathrm{~L}$ & Precentral Gyrus \\
\hline 24 & 4.92 & -55 & $10 \quad 35$ & $\mathrm{~L}$ & Middle Frontal Gyrus \\
\hline 13 & 5.38 & -51 & 720 & $\mathrm{~L}$ & Inferior Frontal Gyrus \\
\hline 12 & 5.01 & -22 & $-37 \quad 70$ & $\mathrm{~L}$ & Postcentral Gyrus \\
\hline 8 & 6.19 & -12 & $-6-15$ & $\mathrm{~L}$ & Parahippocampal Gyrus \\
\hline 2728 & 10.49 & 31 & $-68-15$ & $\mathrm{RC}$ & Declive \\
\hline 25 & 5.61 & 14 & $-59-45$ & $\mathrm{RC}$ & Cerebellar Tonsil \\
\hline 24 & 6.55 & -20 & $-48-15$ & $\mathrm{LC}$ & Culmen \\
\hline 12 & 5.64 & -37 & $-46-40$ & $\mathrm{LC}$ & Cerebellar Tonsil \\
\hline
\end{tabular}

Note: R: right cerebral / L: left cerebral, RC: right cerebellar / LC: left cerebellar

regions of the brain. In particular, large increases were seen in the precuneus, inferior parietal lobule, supramarginal gyrus, middle frontal gyrus, cuneus, and declive.

Table 2 and Figure 2(b) show the activated regions from the "DWT-DO" contrast, i.e., activation during distracted driving was subtracted from the activated regions during driving only. These results indicate an increased activation in the limbic (cingulate gyrus, parahippocampal gyrus, and posterior cingulate) and sub-lobar (lentiform nucleus, caudate, and thalamus) regions of the brain. In particular, large activation increases were observed in the cingulate gyrus, lentiform nucleus, and caudate.

\section{Discussion and conclusion}

The objective of this study was to observe the brain activity when participants were driving and driving while performing distraction tasks, to determine if there were differences between the two 


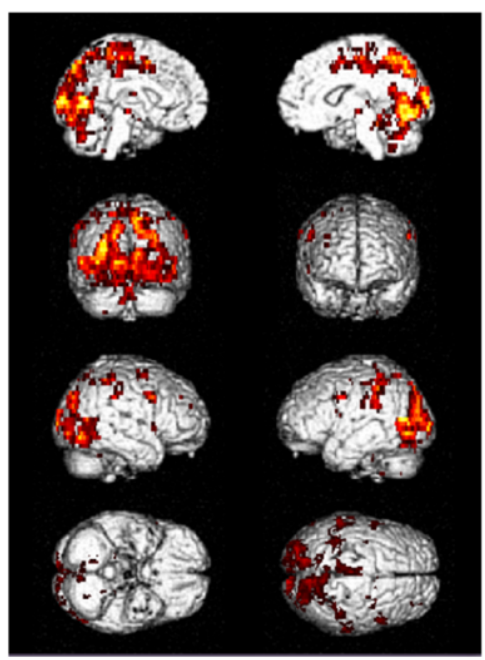

(a)

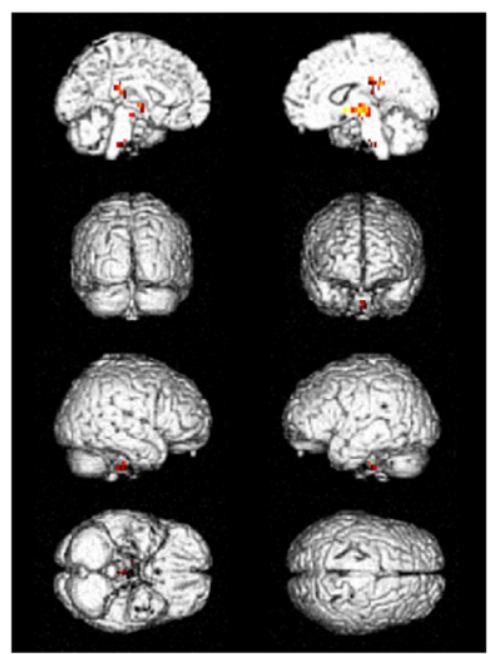

(b)

Fig. 2. The activated regions in the brain, determined by (a) DO-DWT and (b) DWT - DO contrasts (corrected p $<0.05$ ).

Table 2

MNI coordinates, t-scores, and number of voxels in activated regions determined by the double subtraction method (DWTDO) (corrected $\mathrm{p}<0.05)$

\begin{tabular}{|c|c|c|c|c|}
\hline Number of voxels & t-score & $\begin{array}{l}\text { MNI coordinates } \\
(\mathrm{x}, \mathrm{y}, \mathrm{z}(\mathrm{mm}))\end{array}$ & Side & Region \\
\hline 62 & 8.14 & $25-36 \quad 25$ & $\mathrm{R}$ & Cingulate Gyrus \\
\hline 47 & 7.45 & $20-1 \quad-5$ & $\mathrm{R}$ & Lentiform Nucleus \\
\hline 47 & 6.19 & $17-29 \quad 30$ & $\mathrm{R}$ & Caudate \\
\hline 23 & 5.62 & $39-26-10$ & $\mathrm{R}$ & Parahippocampal Gyrus \\
\hline 6 & 5.26 & $5-28 \quad 15$ & $\mathrm{R}$ & Thalamus \\
\hline 22 & 5.98 & $-5-26 \quad 15$ & $\mathrm{~L}$ & Thalamus \\
\hline 20 & 6.21 & $\begin{array}{lll}-23 & -6 & -5\end{array}$ & $\mathrm{~L}$ & Lentiform Nucleus \\
\hline 13 & 4.96 & $-11-34 \quad 20$ & $\mathrm{~L}$ & Posterior Cingulate \\
\hline
\end{tabular}

Note: R: right cerebral / L: left cerebral

conditions. In previous studies where a joystick, computer mouse or a trackball was used for driving, the following regions were all reported as being active: the parietal lobe and precuneus region responsible for spatial perception [7,10]; the cerebellum region for motor control and action planning [3-6,10]; and the cingulate gyrus region for attention and error monitoring $[4,5,7,10]$.

The brain areas activated during DWT phase were similar to those activated during DO phase; however, activation intensity was higher during the DO phase [6]. Just et al. reported that the number of active voxels greatly increased in the supramarginal gyrus, superior and inferior parietal lobes, and superior occipital gyrus when drivers focused on driving only, compared with when they drove while performing an auditory language comprehension distraction task. The results of this study show that the supramarginal gyrus, inferior parietal lobule, and precuneus, which are responsible for spatial perception, had increased activation during the DO condition compared with the DWT condition, 
similar to previous findings $[2,6]$. When the subject performed the DO task, the following regions showed clear activation: the middle frontal gyrus, which is involved in spatial attention and movement planning and execution [7,8]; the cuneus, which is related to visual processing [4]; and the declive, which is related to motor control $[4,6,7]$ (Table 1). The negative effects on these regions, or decreases in activation during distracted driving, are thought to occur because performing distraction tasks interfere with information processing that is related to driving.

The cingulate gyrus and sub-lobar region (lentiform nucleus and caudate), in particular, displayed increased activation during DWT compared with DO (Table 2). These results can be attributed to the fact that when driving is paired with a distraction task, driving performance is affected. Moreover, the increase in desire to control driving performance increases activation in the cingulate gyrus and sublobar region, which are related to error monitoring and unnecessary movement control, respectively $[5,7,13,14]$.

In conclusion, compared with driving only, distracted driving increases activation of the regions associated with error monitoring and unnecessary movement control, and reduces activation of regions associated with driving. Unlike the simple driving simulators with the use of joysticks, computer mice or trackballs in previous research, the addition of a driving wheel and pedals (accelerator and brake) to the driving simulator employed in this study presents a closer real-life driving environment Thus, a more detailed difference in brain activation between the two conditions is observed.

\section{Acknowledgement}

This work was supported by Konkuk University.

\section{References}

[1] M.A. Just, P.A. Carpenter, T.A. Keller, L. Emery, H. Zajac and K.R. Thulborn, Interdependence of nonoverlapping cortical systems in dual cognitive tasks, NeuroImage 14 (2001), 417-426.

[2] M.A. Just, T.A. Keller and J. Cynkar, A decrease in brain activation associated with driving when listening to someone speak, Brain Research 1205 (2008), 70-80.

[3] H. Walter, S.C. Vetter, J. Grothe, A.P. Wunderlich, S. Hahn and M. Spitzer, The neural correlates of driving, NeuroReport 12 (2001), 1763-1767.

[4] V.D. Calhoun, J.J. Pekar, V.B. McGinty, T. Adali, T.D. Watson and G.D. Pearlson, Different activation dynamics in multiple neural systems during simulated driving, Human Brain Mapping 16 (2002), 158-167.

[5] Y. Uchiyama, K. Ebe, A. Kozato, T. Okada and N. Sadato, The neural substrates of driving at a safe distance: a functional MRI study, Neuroscience Letters 352 (2003), 199-202.

[6] Y. Uchiyama, H. Toyoda, H. Sakai, D. Shin, K. Ebe and N. Sadato, Suppression of brain activity related to a carfollowing task with an auditory task: an fMRI study, Transportation Research Part F 15 (2012), 25-37.

[7] F.X. Graydon, R. Young, M.D. Benton, R.J. Genik II, S. Posse, L. Hsieh and C. Green, Visual event detection during simulated driving: identifying the neural correlates with functional neuroimaging, Transportation Research Part F 7 (2004), 271-286.

[8] D. Tomasi, T. Ernst, E.C. Caparelli and L. Chang, Practice-induced changes of brain function during visual attention: a parametric fMRI study at 4 Tesla, NeuroImage 23 (2004), 1414-1421.

[9] S.D. Newman, T.A. Keller and M.A. Just, Volitional control of attention and brain activation in dual task performance, Human Brain Mapping 28 (2007), 109-117.

[10] H.J. Spiers and E.A. Maguire, Neural substrates of driving behavior, NeuroImage 36 (2007), $245-255$.

[11] L. Hsieh, R.A. Young, S.M. Bowyer, J.E. Maran, R.J. Genik II, C.C. Green, Y.J. Chiang, C.C. Liao and S. Seaman, Conversation effects on neural mechanisms underlying reaction time to visual events while viewing a driving scene: fMRI analysis and asynchrony model, Brain Research 1251 (2009), 162-175.

[12] J. Talairach and P. Tournoux, Co-Planar Stereotaxic Atlas of the Human Brain, Thieme, New York, 1998. 
[13] F.G. Flynn, D.F. Benson and A. Ardila, Anatomy of the insula: functional and clinical correlates, Aphasiology 13 (1999), 55-78.

[14] V. Menon, N.E. Adleman, C.D. White, G.H. Glover and A.L. Reiss, Error-related brain activation during a Go/NoGo response inhibition task, Human Brain Mapping 12 (2001), 131-143. 